Review

\title{
A Systematic Approach to Consistent Truncations of Supergravity Theories
}

\section{Michela Petrini}

check for

updates

Citation: Petrini, M. A Systematic Approach to Consistent Truncations of Supergravity Theories. Universe 2021, 7, 485. https://doi.org/ 10.3390/universe7120485

Academic Editor: Norma G. Sanchez

Received: 7 November 2021

Accepted: 30 November 2021

Published: 9 December 2021

Publisher's Note: MDPI stays neutral with regard to jurisdictional claims in published maps and institutional affiliations.

Copyright: (C) 2021 by the author. Licensee MDPI, Basel, Switzerland. This article is an open access article distributed under the terms and conditions of the Creative Commons Attribution (CC BY) license (https:// creativecommons.org/licenses/by/ $4.0 /)$.
Laboratoire de Physique Théorique et Hautes Energies, Sorbonne Université, 4 Place Jussieu, 75005 Paris, France; petrini@lpthe.jussieu.fr

\begin{abstract}
Exceptional generalised geometry is a reformulation of eleven/ten-dimensional supergravity that unifies ordinary diffeomorphisms and gauge transformations of the higher-rank potentials of the theory in an extended notion of diffeormorphisms. These features make exceptional generalised geometry a very powerful tool to study consistent truncations of eleven/ten-dimensional supergravities. In this article, we review how the notion of generalised G-structure allows us to derive consistent truncations to supergravity theories in various dimensions and with different amounts of supersymmetry. We discuss in detail the truncations of eleven-dimensional supergravity to $\mathcal{N}=4$ and $\mathcal{N}=2$ supergravity in five dimensions.
\end{abstract}

Keywords: string compactifications; generalised geometry; supergravity

\section{Introduction}

A central problem in string theory is how to derive lower-dimensional effective theories describing the universe we observe.

String theory is our best candidate for a unified description of all fundamental forces, but at the price of a universe with ten (or eleven) space-time dimensions. To make contact with observations, one considers solutions of string theory where the space-time is the product of a non-compact space-time $X$ and a compact manifold $M$, which is too small to be observed.

The fluctuations around such solutions can be organised as particles in $X$ whose properties depend on the geometry of the internal manifold $M$. In the same way as the Fourier expansion on a circle gives an infinite set of modes, the expansion of string fluctuations on the internal manifold $M$ gives an effective low energy theory with an infinite set of modes in $X$, the Kaluza-Klein towers.

The question is then how to truncate the theory to a finite set of modes so that there is no coupling between the modes that are kept and those that are discarded. In some cases, such as compactifications on special holonomy manifolds, there is a clear notion of light (massless) and heavy modes, and the effective theory is obtained by keeping only the massless ones. In other cases, such as Anti de Sitter compactifications, there is no natural separation between light and heavy modes, and a truncation procedure is required.

A consistent truncation is a procedure to truncate the Kaluza-Klein states to a finite set in such a way that the dependence of the higher-dimensional fields on the internal manifold factorises out once the truncation ansatz is plugged in the equations of motion. This condition is what makes consistent truncations relatively rare and hard to prove (see, for instance $[1,2])$.

Typically, a consistent truncation relies on the geometrical properties of the compactification manifold. The best known examples are Scherk-Schwarz reductions, where the internal space is a group manifold $\mathcal{G}$ (or a quotient $\mathcal{G} / \Gamma$ by a freely acting discrete group Г) [3], and consistency is a consequence of keeping only modes invariant under the group action. However, there are examples, such as the reductions of eleven-dimensional super- 
gravity on $S^{7}[4]$ and on $S^{4}$ [5], where the consistency is not a consequence of any manifest symmetry.

In the last few years, reformulations of ten/eleven-dimensional supergravities such as exceptional generalised geometry and exceptional field theory have considerably improved the situation, and now we have a framework to systematically study consistent truncations in different dimensions and with different amounts of supersymmetry. For instance, all maximally supersymmetric truncations, conventional Scherk-Schwarz reductions as well as sphere truncations, are interpreted as generalised Scherk-Schwarz reductions [6-11]. Thanks to this interpretation, it was possible to prove the long-standing conjecture of the consistency of type IIB supergravity on $S^{5}[6,10,12]$ and to reproduce $[13,14]$ maximally supersymmetric truncations of massive type IIA supergravity [15-17].

Truncations to half-maximal supergravities have also been explored rather extensively [18-23], while $\mathcal{N}=2$ truncations have been studied in [24]. This approach also allows one to give a proof [23] of the conjecture in [25] that given any supersymmetric solution of ten/eleven-dimensional supergravity of the form $A d S_{D} \times M$, one can construct a consistent truncation to pure gauged supergravity in $D$ dimensions containing that solution and having the same supersymmetry.

In this article, we will review the exceptional generalised geometry approach to consistent truncations. Exceptional generalised geometry provides a unified geometrical interpretation of ordinary diffeomorphisms and gauge transformations of the higherrank potentials of eleven/ten-dimensional supergravities as generalised diffeomorphisms. This is achieved by replacing the tangent bundle to a manifold $M$ with a larger one, the generalised tangent bundle, whose fibres transform as representations of the U-duality group. In this language, the key notion to study consistent truncations is that of the generalised $G_{S}$-structure, namely the reduction of the structure group of the generalised tangent bundle by nowhere vanishing generalised tensors on $M$. In [23], it was proved that given a manifold $M$ admitting a generalised $G_{S}$-structure with singlet intrinsic torsion, a consistent truncation of any field theory on $M$ is obtained by expanding all the fields on the $G_{S}$ invariant tensors and keeping only those transforming as singlets.

In this language, all maximally supersymmetric truncations correspond to generalised parallelisable manifolds, namely to a generalised identity structure, while truncations preserving less supersymmetry are based on generalised structures larger than the identity. In all cases, the data of the generalised $G_{S}$-structure are enough to determine all the features of the lower-dimensional gauged supergravity: amount of supersymmetry, field content, and the gaugings.

As a generalised $G_{S}$-structure does not always correspond to an ordinary one, this approach considerably enlarges the space of consistent truncations. In fact, all consistent truncations of higher-dimensional supergravities around solutions of the type $X \times M$, where $M$ is a Riemannian manifold of dimension $d \leq 7$, should be described by generalised $G_{S}$-structures.

This paper is organised as follows. In Section 2, we will recall the basic notions of ordinary $G_{S}$-structures and how they are related to consistent truncations, while Section 3 contains the extension of these ideas to exceptional geometry. We will briefly discuss the example of the generalised Scherk-Schwarz reduction and then show how this approach allows us to prove the conjecture of [25] that any supersymmetric solution to ten/elevendimensional supergravity that is a warped product of $A d S_{D} \times M$ admits a consistent truncation to pure gauged supergravity in $D$ dimensions containing that solution and having the same amount of supersymmetry.

Since the formalism is based on the exceptional U-duality groups, the details of the truncation depend on the dimension of the internal manifold $M$. In Section 4, we focus on truncations of eleven-dimensional supergravity giving rise to $\mathcal{N}=4$ and $\mathcal{N}=2$ five-dimensional theories. Rather than describing explicit examples of truncations, which can be found in $[23,24]$, we will discuss the general procedure and how the data of the $G_{S}$-structure on the internal manifold are mapped onto those of the truncated theory. 


\section{Conventional G-Structures and Consistent Truncations}

Before moving to generalised geometry, it is instructive to review what a conventional $G_{S}$-structure is and how it is related to consistent truncations.

A $d$-dimensional manifold $M$ has a $G_{S}$-structure if its structure group is reduced to $G_{S} \subset G L(d, \mathbb{R})$. The $G_{S}$-structure is defined by a set of $G_{S}$-invariants, nowhere vanishing tensors $\left\{\Xi_{i}\right\}^{1}$. For example, an invariant metric tensor $g$ or, equivalently, a subset of orthonormal frames on $M$ defines a $G_{S}=O(d)$ structure. This also implies that for Riemannian manifolds, the possible $G_{S}$-structures are all subgroups of $O(d)$.

Any $G_{S}$-structure is characterised by its intrinsic torsion. For Riemannian manifolds, the intrinsic torsion can be defined via the action of the Levi-Civita connection on the invariant tensors $\Xi_{i}$ :

$$
\begin{aligned}
& \nabla_{m} \Xi_{i}^{{ }^{n_{1} \ldots n_{r}}} p_{1} \ldots p_{s}=K_{m}{ }^{n_{1}}{ }_{q} \Xi_{i}{ }^{q} \ldots n_{r} p_{1} \ldots p_{s}+\cdots+K_{m}{ }^{n_{r}}{ }_{q} \Xi_{i}{ }^{n_{1} \ldots q} p_{1} \ldots p_{s} \\
& -K_{m}{ }^{q} p_{1} \Xi_{i}{ }^{n_{1} \ldots n_{r}} q_{\ldots p_{s}}+\cdots-K_{m}{ }^{q} p_{s} \Xi_{i}{ }^{n_{1} \ldots n_{r}} p_{1} \ldots q \text {. }
\end{aligned}
$$

The tensor $K_{m}{ }^{n} p$ is a section of $T^{*} M \otimes \Lambda^{2} T^{*} M$, where the indices $m$ and $n, p$ span $T^{*} M$ and $\Lambda^{2} T^{*} M$, respectively. Decomposing $\Lambda^{2} T^{*} M \simeq S O(d)=\mathfrak{g} \oplus \mathfrak{g}^{\perp}$, where $\mathfrak{g}$ is the Lie algebra of $G_{S}$, and using the fact that $\Xi_{i}$ are $G_{S}$-invariant, we see that $K$ is actually a section of $T^{*} M \otimes \mathfrak{g}^{\perp}$.

The intrinsic torsion is defined as

$$
\left(T_{\text {int }}\right)_{m n}{ }^{p}=K_{n}{ }^{p}{ }_{m}-K_{m}{ }^{p}
$$

and gives the part of the torsion that does not depend on the choice of connection. $T_{\text {int }}$ can be decomposed into $G_{S}$ representations, known as the "torsion classes" of the structure. For consistent truncations, we are interested in $G_{S}$-structures whose non-zero torsion components are constant singlets under $G_{S}$.

A series of papers showed that $G$-structures are powerful tools to study consistent truncations [26-32].

Suppose a $d$-dimensional manifold $M$ admits a $G_{S}$-structure defining a set of invariant tensors $\Xi_{i}$, with $G_{S} \supset O(d)$ and only constant, singlet intrinsic torsion. Then, a field theory can be consistently truncated on $M$ by expanding all the fields on the basis of tensors $\Xi_{i}$, which encode the dependence on the internal space, and only keeping the fields that are $G_{S}$ singlets. Since the intrinsic torsion has only singlet components, (1) implies that the derivatives of the singlet fields can only contain singlets. Thus, the truncation is necessarily consistent, since products of singlet representations can never source the non-singlet representations that were truncated away.

If the theory includes spinors, the $G_{S}$-structure lifts to a $\tilde{G}_{S} \subset \operatorname{Spin}(d)$ structure, and we simply have to expand the spinor fields in terms of the spinors invariant under $\tilde{G}_{S}$.

The data of the $G_{S}$-structure also determine the field content and gauge interactions of the truncated theory.

For instance, we can easily determine the scalar and vector fields coming from the reduction of the higher-dimensional metric. The scalars are the $G_{S}$ singlet components of the metric. Since the metric parameterises the coset $G L(d, \mathbb{R}) / O(d)$, these are given by the $G L(d, \mathbb{R})$ deformations of a reference metric that commute with the $G_{S}$ modulo, the $O(d)$ deformations that commute with $G_{S}$ :

$$
\text { metric scalars } \Leftrightarrow H \in \frac{\operatorname{Com}_{G L(d, \mathbb{R})}\left(G_{S}\right)}{\operatorname{Com}_{O(d)}\left(G_{S}\right)},
$$

where $\operatorname{Com}_{B}(A)$ denotes the commutant of the subgroup $A$ of $B$ inside $B$.

The vectors coming from the metric are given by the $G_{S}$-invariant one-forms $\eta^{a} \in\left\{\Xi_{i}\right\}$. If we call $\hat{\eta}_{a}$ the singlet vectors dual to $\eta^{a}$, we have

$$
\text { metric gauge fields } \Leftrightarrow A_{\mu}^{a} \hat{\eta}_{a} \text {. }
$$


The components of the singlet intrinsic torsion are completely determined by the Lie derivatives of the invariant tensors

$$
\mathcal{L}_{\hat{\eta}_{a}} \Xi_{i}=f_{a i}{ }^{j} \Xi_{j}
$$

where $f_{a i}{ }^{j}$ are constants. They also give the gauge algebra of the metric gauge fields via the Lie bracket

$$
\left[\hat{\eta}_{a}, \hat{\eta}_{b}\right]=f_{a b}^{c} \hat{\eta}_{c}
$$

Conventional Scherk-Schwarz reductions on a group manifold $M=\mathcal{G}$ can be reinterpreted in this language. The group manifold admits a basis of globally defined (left-invariant) one-forms, $\left\{e^{a}\right\} \in T^{*} M$, which reduce the structure group to $G_{S}=\mathbb{I}$.

The scalar fields of the truncated theory parameterise the coset

$$
\frac{\operatorname{Com}_{G L(d)}(\mathbb{I})}{\operatorname{Com}_{S O(d)}(\mathbb{I})}=\frac{G L(d, \mathbb{R})}{S O(d)} .
$$

The Maurer-Cartan equations

$$
\mathrm{d} e^{a}=f_{b c}{ }^{a} e^{b} \wedge e^{c}
$$

with $f_{b c}{ }^{a}$ structure constants of the Lie algebra $\mathfrak{g}$, imply that the identity structure has singlet, constant intrinsic torsion since the exterior derivative of the invariant one-forms are also expressed on the $e^{a}$ basis, and the coefficients of the expansion are constant.

The one-forms define $d$ gauge fields with a Lie algebra given by the Lie bracket (6). The consistent truncation ansatz for the metric is

$$
\mathrm{d} s^{2}=g_{\mu \nu} \mathrm{d} x^{\mu} \mathrm{d} x^{\nu}+h_{a b}\left(e^{a}+A^{a}\right)\left(e^{b}+A^{b}\right),
$$

where $h_{a b}(x)$ is a matrix of scalar fields, and $A_{\mu}^{a}(x)$ are gauge fields in the adjoint of $G_{S}$.

Another interesting example is the reduction of M-theory and type IIB on a SasakiEinstein manifold $M$ of dimension $d=2 n+1[26,29,30]$. The manifold admits a $G_{S}=S U(n)$ structure defined by a real one-form $\eta$, a real two-form $\omega$, and a complex $n$-form $\Omega$, satisfying

$$
\mathrm{d} \eta=2 \omega, \quad \mathrm{d} \Omega=i(n+1) \eta \wedge \Omega .
$$

Since only invariant tensors appear on the right-hand side of the differential conditions (10), the intrinsic torsion has only constant singlet components. In this case, the metric scalar manifold is

$$
\frac{\operatorname{Com}_{G L(2 n+1, \mathbb{R})}(S U(n))}{\operatorname{Com}_{S O(2 n+1)}(S U(n))}=\frac{\mathbb{R}^{+} \times \mathbb{C}}{U(1)}=\mathbb{R}^{+} \times \mathbb{R}^{+} .
$$

As there is a single invariant one-form $\eta$, the truncated theory will contain only one gauge field $A_{\mu}(x)$ coming from the metric. The ansatz for the metric is

$$
\mathrm{d} s^{2}=g_{\mu \nu} \mathrm{d} x^{\mu} \mathrm{d} x^{v}+e^{2 U} \mathrm{~d} s_{2 n}^{2}+e^{2 V}(\eta+A)^{2}
$$

where $\mathrm{d} s_{2 n}^{2}$ is the (local) $2 n$-dimensional Kähler-Einstein metric defined by $(\omega, \Omega)$. The scalar fields $U(x)$ and $V(x)$ parametrise the scalar manifold.

\section{Generalised G-Structures and Consistent Truncations}

The approach based on conventional $G_{S}$-structures have allowed several examples of consistent truncations to be constructed [26-32], but there are other well-known examples that do not admit such a description. This is the case, for instance, of maximally supersymmetric consistent truncations on spheres, such as eleven-dimensional supergravity on $S^{7}[4]$ and $S^{4}[5]$. 
By extending the notion of the $G_{S}$-structure, exceptional generalised geometry $[33,34]$ allows these examples to be treated on the same footing as the conventional ScherkSchwarz reductions, and more generally, it provides a new systematic way to study consistent truncations with a generic amount of supersymmetry: reducing a supergravity theory on any manifold $M$ admitting a generalised $G_{S}$-structure with constant singlet intrinsic torsion gives a consistent truncation [23].

In this section, we will give the main ideas without entering into the details of a specific theory or compactification. If the discussion is too vague, the reader can skip to the next section where truncations of M-theory to five dimensions are described in more detail.

Exceptional generalised geometry replaces the tangent bundle $T M$ with a larger bundle $E$ on $M$, whose fibres transform in a representation of the exceptional group $E_{d(d)}$. In this way, the diffeomorphisms and gauge symmetries of higher-dimensional supergravity are unified as generalised diffeomorphisms on $E$. Then, one can generalise all conventional notions of differential geometry such as tensors, connections, and Lie derivatives.

The bundle $E$ is called the generalised tangent bundle, and its sections are generalised vectors. The dual generalised vectors are sections of the bundle $E^{*}$, and generalised tensors are obtained by tensoring $E$ and/or $E^{*}$. For example, we will need the dual vectors bundle $Z_{b}$, which are sections of the bundle ${ }^{2} N \sim \operatorname{det} T^{*} M \otimes E^{*}$, and the generalised metric, which is a section of the symmetric product $S^{2}\left(E^{*}\right)$. In analogy with an ordinary metric on $M$, a generalised metric $G$ parameterises, at each point on $M$, the coset

$$
G \in \frac{E_{d(d)}}{H_{d}},
$$

where $H_{d}$ is the maximally compact subgroup of $E_{d(d)}$. Spinors can also be introduced as sections of the spinor bundle $\mathcal{S}$, transforming in the spinorial representation of $\tilde{H}_{d}$, the double cover of the group $H_{d}$.

The action of an infinitesimal generalised diffeomorphism is generated by the generalised Lie derivative along a generalised vector. We denote by adj $F$ the adjoint bundle, namely the bundle whose fibres transform in the adjoint of $E_{d(d)}$. Then, in analogy with the conventional Lie derivative, we define the generalised one as an adjoint $E_{d(d)}$ action [35],

$$
\left(L_{V} V^{\prime}\right)^{M}=V^{N} \partial_{N} V^{M}-\left(\partial \times{ }_{\text {adj }} V\right)^{M}{ }_{N} V^{\prime N},
$$

where $V^{M}$ are the components of the generalised vector $V$ in a standard coordinate basis, $\partial_{M}=\partial_{m}$ are viewed as sections of the dual tangent bundle, and the projection onto the adjoint bundle is $\times_{\text {adj }}: E^{*} \otimes E \rightarrow \operatorname{adj} F$.

The definition of a generalised $G_{S}$-structure is a natural extension of the conventional one. A generalised $G_{S}$-structure on $M$ is the reduction of the generalised structure group $E_{d(d)}$ to a subgroup $G_{S}$, and it is defined by a set of nowhere vanishing $G_{S}$-invariant generalised tensors $\left\{Q_{i}\right\}$. For instance, the generalised metric defines a $G_{S}=H_{d}$ structure on $M[35,36]$. In what follows, we will always assume that $M$ admits an $H_{d}$ structure, and we will always consider generalised structures $G_{S} \subset H_{d}$.

Given a generalised $G_{S}$-structure, with $G_{S} \subseteq H_{d}$, defined by a set of $G_{S}$-invariant generalised tensors $\left\{Q_{i}\right\}$, we can define its intrinsic torsion from the Lie derivative of a generalised tensor $\alpha$ along a generalised vector $V$ [37]:

$$
\left(L_{V}^{\tilde{D}}-L_{V}\right) \alpha=T(V) \cdot \alpha .
$$

Here, $L_{V}$ is the generalised Lie derivative defined in (14), and $L_{V}^{\tilde{D}}$ is the generalised Lie derivative calculated using a $G_{S}$-compatible connection ${ }^{3} \tilde{D}$. The torsion can be seen as a map from the generalised tangent bundle into the adjoint one, $T: \Gamma(E) \rightarrow \Gamma(\operatorname{adj} F)$, so that $T(V)$ acts on $\alpha$ via the adjoint action. 
The intrinsic torsion $T_{\text {int }}$ is then the component of $T$ that is independent of the choice of compatible connection $\tilde{D}$ and can be decomposed into representations of $G_{S}$.

Consider now eleven-dimensional or type II supergravity on a product space $X \times M$, where $X$ is a $D$-dimensional Lorentzian space, and $M$ is an internal manifold of dimension $d$ in M-theory and $d-1$ in type II supergravity. We assume $d \leq 7$.

As we discussed above, the $G L(d, \mathbb{R})$ or $G L(d-1, \mathbb{R})$ structure groups of conventional geometry on $M$ are extended to $E_{d(d)}$. The idea is then to rearrange the supergravity fields into generalised tensors transforming as representations of $G L(D, \mathbb{R}) \times E_{d(d)}$ and to interpret the theory as a $D$-dimensional theory on $X$ with an infinite number of fields. The fields in $X$ will be scalar, vectors, and two-forms according to their $G L(D, \mathbb{R})$ representation ${ }^{4}$.

The scalar degrees of freedom on $X$ are given by the components of all supergravity fields (metric and higher-rank potentials) with all internal indices and are repackaged into a generalised metric. The $G L(D, \mathbb{R})$ one-forms and vectors are sections of the generalised tangent space $E$, while the two-forms are sections of the bundle $N$. In summary, we have

$$
\begin{aligned}
\text { scalars: } & G_{M N}(x, y) & \in \Gamma\left(S^{2} E^{*}\right), \\
\text { vectors: } & \mathcal{A}_{\mu}{ }^{M}(x, y) & \in \Gamma\left(T^{*} X \otimes E\right), \\
\text { two-forms: } & \mathcal{B}_{\mu \nu}{ }^{M N}(x, y) & \in \Gamma\left(\Lambda^{2} T^{*} X \otimes N\right),
\end{aligned}
$$

where $x$ and $y$ are coordinates on $X$ and $M$, respectively, and the capital index $M$ denotes components of vectors in $E$ or $E^{*}$.

In Table 1, we list the exceptional group and the representations for the generalised vectors $(E)$, their weighted duals $(N)$, the adjoint, and the spinor bundle $S$, in which the supersymmetry parameter lies [36], for different dimensions of the non-compact space $X$.

Table 1. Generalised geometry groups, bundles, and representations.

\begin{tabular}{ccccccc}
\hline $\boldsymbol{D}$ & $\boldsymbol{E}_{\boldsymbol{d}(\boldsymbol{d})}$ & $\boldsymbol{E}$ & ad $\boldsymbol{F}$ & $\boldsymbol{N}$ & $\tilde{\boldsymbol{H}}_{\boldsymbol{d}}$ & $\mathcal{S}$ \\
\hline 4 & $E_{7(7)}$ & $\mathbf{5 6}$ & $\mathbf{1 3 3}$ & $\mathbf{1 3 3}$ & $S U(8)$ & $\mathbf{8} \oplus \overline{\mathbf{8}}$ \\
5 & $E_{6(6)}$ & $\mathbf{2 7}$ & $\mathbf{7 8}$ & $\mathbf{2 7 ^ { \prime }}$ & Usp $(8)$ & $\mathbf{8}$ \\
6 & $S p i n(5,5)$ & $\mathbf{1 6}$ & $\mathbf{4 5}$ & $\mathbf{1 0}$ & Usp $(4) \times U s p(4)$ & $(\mathbf{4}, \mathbf{1}) \oplus(\mathbf{1}, \mathbf{4})$ \\
7 & $S L(5, \mathbb{R})$ & $\mathbf{1 0}$ & $\mathbf{2 4}$ & $\mathbf{5}^{\prime}$ & Usp $(4)$ & $\mathbf{4}$ \\
\hline
\end{tabular}

The equations of motion and the supersymmetry variations are also organised according to the representations above, and the dynamics of the supergravity is completely determined by the Levi-Civita connection on the external space $X$ and a generalised connection on $M$.

If the manifold $M$ has a generalised $G_{S}$-structure, $G_{S} \subset H_{d}$, with only constant, singlet intrinsic torsion, we can construct a consistent truncation in the following way. Expand all bosonic fields in terms of the $G_{S}$ invariant tensors $\left\{Q_{i}\right\}$ defining the structure, and keep only those transforming as singlets under the structure group. The coefficient of the expansion will depend on the external coordinates $x$, while the dependence on the internal space is only in the tensors $\left\{Q_{i}\right\}$.

Since there are only singlet representations in the intrinsic torsion, the generalised Levi-Civita connection acts on any invariant generalised tensor $Q_{i}$ as

$$
D_{M} Q_{i}=\Sigma_{M} \cdot Q_{i}
$$

where $\Sigma_{M}$ is a section of $E^{*} \otimes \operatorname{adj}\left(H_{d}\right)$ that is completely determined in terms of the constant singlet torsion. Here, $\operatorname{adj}\left(H_{d}\right)$ denotes the bundle of tensors transforming in the adjoint representation of $H_{d}$. This means the derivatives of all the truncated fields are also expanded in terms of singlets only. Since products of singlet representations cannot source non-singlet representations, keeping only all possible singlets gives a consistent truncation. 
To extend the truncation to the fermionic sector of the supergravity theory, it is enough to lift the structure group $G_{S}$ to $\tilde{G}_{S} \subset \tilde{H}_{d}$ and to expand all the fermionic fields in terms of $\tilde{G}_{S}$ singlets.

From the data of the $G_{S}$-structure, we can determine the number of scalars, vectors, one-forms, and two-forms of the truncated theory, as well as the possible gaugings.

All scalars of the truncated theory are given by the $G_{S}$ singlets in the generalised metric $G_{M N}$. These are singlet deformations of the structure modulo, those singlet deformations that do not deform the metric

$$
\text { scalars: } \quad h^{I}(x) \in \mathcal{M}=\frac{\operatorname{Com}_{E_{d(d)}}\left(G_{S}\right)}{\operatorname{Com}_{H_{d}}\left(G_{S}\right)}=\frac{\mathcal{G}}{\mathcal{H}} .
$$

Consider now the vectors of the truncated theory. Being sections of $T^{*} X \otimes E$, they are determined by the number of $G_{S}$ invariant generalised vectors $\left\{K_{I}\right\}$ :

$$
\text { vectors: } \quad \mathcal{A}_{\mu}^{M}(x, y)=A_{\mu}^{I}(x) \mathcal{K}_{I}^{M} \in \Gamma\left(T^{*} M \otimes \mathcal{V}\right),
$$

where $\mathcal{V} \subset \Gamma(E)$ is the vector space spanned by the $\left\{\mathcal{K}_{I}\right\}$.

Similarly the two-forms are determined by the $G_{S}$ singlets in the bundle $N$ :

$$
\text { two-forms: } \quad \mathcal{B}_{\mu \nu}{ }^{M N}(x, y)=\mathcal{B}_{\mu \nu}(x) K_{b}^{I M N} \in \Gamma\left(\Lambda^{2} T^{*} X \otimes \mathcal{B}\right),
$$

where $\left\{K_{b}^{I}\right\}$ is a basis generating the $G_{S}$-invariant vector space $\mathcal{B} \subset \Gamma(N)$.

Let us stress again that the representations above determine the full content of the theory, namely the fields coming from the reduction of the metric and the higher-rank potentials of the supergravity theory. In particular, this means that the vectors $K_{I}$ generate all symmetries of the reduced theories, coming both from the metric and the higher-rank potentials. This is an important difference with respect to the reductions based on the conventional $G_{S}$-structure.

The $G_{S}$-structure also determines the embedding tensor (see $[40,41]$ for a review of this formalism) and hence the gaugings of the reduced theory in terms of the singlet intrinsic torsion.

Since the $G_{S}$-structure has only singlet intrinsic torsion, in analogy with (5), the generalised Lie derivative of the $G_{S}$-invariant generalised tensors along any invariant generalised vector $K_{I}$ can be written as

$$
L_{K_{I}} Q_{i}=-T_{\text {int }}\left(\mathcal{K}_{I}\right) \cdot Q_{i},
$$

where $T_{\text {int }}$ now maps the space $\mathcal{V}$ of the $G_{S}$ invariant vector to the $G_{S}$ singlets in the adjoint bundle. This means that $T_{\text {int }}\left(\mathcal{K}_{I}\right)$ must correspond to the elements in the adjoint that commute with $G_{S}$, namely the Lie algebra of the commutant group $\mathcal{G}=\operatorname{Com}_{E_{d(d)}}\left(G_{S}\right)$. $\mathcal{G}$ is the subgroup of the isometry group of the scalar manifold that can a priori be gauged in the truncated theory.

Since $T_{\text {int }}$ defines a linear map from the space of $G_{S}$ singlet vectors to the Lie algebra Lie $\mathcal{G}$, we can identify $-T_{\text {int }}$ with the embedding tensor of the truncated theory

$$
\Theta: \mathcal{V} \rightarrow \operatorname{Lie} \mathcal{G}
$$

The generalised Lie derivative among the $G_{S}$-invariant vectors gives

$$
L_{K_{I}} K_{J}=\Theta_{I} \cdot \mathcal{K}_{J}=\Theta_{I}{ }^{\hat{\alpha}}\left(t_{\hat{\alpha}}\right)_{J}{ }^{K} \mathcal{K}_{K}:=X_{I J}{ }^{K} \mathcal{K}_{K},
$$

where $\left(t_{\hat{\alpha}}\right)_{J}{ }^{K}$ are the representations of the generators of LieG acting on $\mathcal{V}$. The Leibniz property of the generalised Lie derivative $[6,35]$ translates into the quadratic condition on the embedding tensor

$$
\left[X_{I}, X_{J}\right]=-X_{I J}{ }^{K} X_{K},
$$


with $\left(X_{I}\right)_{J}^{K}=X_{I J}{ }^{K}$ a matrix.

Thus, the generalised vectors $\mathcal{K}_{I}$ generate a Lie algebra with structure constants $X_{[I J]}{ }^{K}$. This is the gauge algebra of the truncated theory. Then, the gauge group is

$$
\text { gauge group: } \quad G_{\text {gauge }} \subseteq \mathcal{G}
$$

Notice that since the image of the map $\Theta$ may not be the whole of Lie $\mathcal{G}$, the gauge group generated by the vectors can be a subgroup of $\mathcal{G}$. The matrices $X_{I}$ then define the adjoint representation, and $\Theta$ defines how the gauge action embeds as an action in $\mathcal{G}$.

The scalar covariant derivatives are

$$
\hat{D}_{\mu} h^{I}=\partial_{\mu} h^{I}-k_{\mu}^{I} \Theta_{I}{ }^{\hat{\alpha}} k_{\hat{\alpha}}^{I},
$$

where $k_{\hat{\alpha}}$ are the Killing vectors on $\mathcal{M}$ generating the action of the Lie $\mathcal{G}$.

The $G_{S}$-structure also determines the fermionic sector of the truncated theory and in particular the number of preserved supersymmetries. Given a lift $\tilde{G}_{s} \subseteq \tilde{H}_{d}$, the number of supersymmetries preserved by the truncated theory is given by the number of $\tilde{G}_{S}$-singlets in the generalised spinor bundle $\mathcal{S}$. Depending on the choice of structure group $G_{S}$, one can construct truncations with different amounts of supersymmetry.

As an example, consider maximally supersymmetric truncations. These are all associated to a generalised identity structure, or generalised parallelisation on the generalised tangent bundle $E$, and can be seen as generalised Scherk-Schwarz reductions [6].

A manifold $M$ is generalised (Leibniz) parallelisable if there exists a globally-defined frame $\left\{E_{A}\right\}$ for the generalised tangent bundle $E$ satisfying the algebra

$$
L_{E_{A}} E_{B}=X_{A B}{ }^{C} E_{C},
$$

with constant coefficients $X_{A B}{ }^{C}$. Notice that the generalised Lie derivative $L$ is not antisymmetric, and therefore the algebra (27) is a Leibniz algebra and not necessarily a Lie algebra. Hence the name Leibniz parallelisation.

Combining (27) and the Leibniz property of the generalised Lie derivative, we see that the constants $X_{A B}^{C}$ realise the gauge algebra

$$
\left[X_{A}, X_{B}\right]=-X_{A B}{ }^{C} X_{C},
$$

where, again, we see $\left(X_{A}\right)_{B}{ }^{C}$ as matrices. Thus, the constants $X_{A B}{ }^{C}$ are the generators of the gauge group.

Starting from a generalised Leibniz parallelisation, one can define a generalised ScherkSchwarz reduction. We define a twisted generalised frame by acting on $\left\{E_{A}\right\}$ with an $E_{d(d)}$ matrix $U_{A}{ }^{B}(x)$ that depends on the external coordinates $x$,

$$
\hat{E}_{A}^{M}(x, y)=U_{A}^{B}(x) E_{B}^{M}(y),
$$

with $M$ denoting the generalised vector components, and then use it to define a generalised (inverse) metric

$$
G^{M N}(x, y)=\delta^{A B} \hat{E}_{A}^{M}(x, y) \hat{E}_{B}^{M}(x, y)=\mathcal{M}^{A B}(x) E_{A}^{M}(y) E_{B}^{M}(y) .
$$

The matrix $M^{A B}=\delta^{C D} U_{C}{ }^{A} U_{D}{ }^{B}$ parameterises the coset $E_{d(d)} / H_{d}$ and contains all the scalars of the lower-dimensional theory.

The frame $\left\{E_{A}\right\}$ also provides the full set of vector fields of the truncated theory

$$
A_{\mu}^{M}(x, y)=A_{\mu}^{A}(x) E_{A}^{M}(y),
$$

and the two-forms are given by

$$
B_{\mu \nu}^{M N}(x, y)=\frac{1}{2} B_{\mu \nu}^{A B}(x)\left(E_{A} \otimes_{N} E_{B}\right)^{M N}(y)
$$


where $\otimes_{N}$ denotes the projection onto the bundle $N$.

The notion of Leibniz parallelisation allows us to go beyond ordinary Scherk-Schwarz reductions to encompass all reductions on coset manifolds. ${ }^{5}$ In particular, as shown in $[6,10,12]$, the consistent truncations of eleven-dimensional supergravity on $S^{7}$ and $S^{4}$ and type IIB supergravity on $S^{5}$ can all be interpreted as generalised Scherk-Schwarz reductions. The crucial observation was that in all these cases, the solutions contain a higher-rank form on the internal sphere that makes it possible to define a nowhere vanishing generalised frame. A similar analysis [14] for massless type IIA allowed for recovering all known maximally supersymmetric truncations on $S^{d}$ with $d=2,3,4,6$, while for massive IIA, one can reproduce the truncation on $S^{6}$ of [15-17] and prove that no maximally supersymmetric truncations are possible for $d=2,3,4$.

We conclude this section with another application of a generalised $G_{S}$-structure to consistent truncation. In [25], it was conjectured that for any solution of a supergravity theory that is a warped product of a $D$-dimensional AdS (or Minkowski) space-time with internal manifold $M$ and preserves $N$ supersymmetries, there is a consistent truncation to a pure supergravity in $D$ dimension with the same amount of supersymmetry. Using generalised $G_{S}$-structures, the proof of the conjecture is very simple. It was observed in $[37,42,43]$ that solutions with an AdS factor and $N$ supersymmetries are associated to generalised $G_{S}$ structures with singlet intrinsic torsion. This is exactly the condition for a solution to admit a consistent truncation, and the truncated theory is pure gauged supergravity [23] (see also [20] for half-maximal truncations). The same argument holds for solutions with a Minkowski factor, the only difference being that the truncated theory is ungauged.

\section{M-Theory Truncations to Five Dimensions}

The construction described in the previous section applies to truncations of supergravity in eleven or ten dimensions on manifolds with dimensions $d \leq 7$ and with any amount of supersymmetry. The exceptional group and, hence, the details of the truncations depend on the dimension $d$ of the internal manifold.

In this section, we focus on compactifications of eleven-dimensional supergravity to five dimensions and $\mathcal{N}=2$ supergravity to five dimensions. Even if we specify, for simplicity, eleven-dimensional reductions to five dimensions, the generalised structures also describe the reduction of type IIB supergravity to five dimensions. The only difference will be in the identification of the generalised tensors with the supergravity fields.

The discussion of this section is based on $[23,24,44]$.

\section{1. $E_{6(6)}$ Generalised Geometry}

We start with a brief review of the generalised geometry describing these compactifications. For more details, see [45].

Compactifications of eleven-dimensional supergravity on a six-dimensional manifold $M$ are described by $E_{6(6)} \times \mathbb{R}^{+}$generalised geometry.

The tangent bundle TM is extended to the generalised tangent bundle $E$ on $M$ whose sections transform in the representation 27 of the generalised structure group $E_{6(6)}$. The structure group of $T M, G L(6, \mathbb{R})$, embeds as the geometrical subgroup of $E_{6(6)}$. The generalised tangent bundle can be written in terms of $G L(6, \mathbb{R})$ representations as

$$
E \simeq T M \oplus \Lambda^{2} T^{*} M \oplus \Lambda^{5} T^{*} M,
$$

and its sections, the generalised vectors, consist, locally, of the sum of a vector, a two-form, and a five-form on $M$,

$$
V=v+\omega+\sigma .
$$

Other generalised tensors are defined as bundles whose fibres transform in different representations of $E_{6(6)}$. The dual generalised vectors are sections of the bundle transforming in the $\overline{\mathbf{2 7}}$ of $E_{6(6)}$, 


$$
E^{*} \simeq T^{*} M \oplus \Lambda^{2} T M \oplus \Lambda^{5} T M,
$$

and are locally sums of a one-form $\hat{v}$, a two-vector $\hat{\omega}$, and a five-vector $\hat{\sigma}$ :

$$
Z=\hat{v}+\hat{\omega}+\hat{\sigma} .
$$

Generalised vectors and dual generalised vectors have a natural pairing,

$$
\langle Z, V\rangle=\hat{v}_{m} v^{m}+\frac{1}{2} \hat{\omega}^{m n} \omega_{m n}+\frac{1}{5 !} \hat{\sigma}^{m n p q r} \sigma_{m n p q r} .
$$

The $E_{6(6)}$ cubic invariant is defined on $E$ and $E^{*}$ as

$$
\begin{aligned}
c(V, V, V) & =-6 l_{v} \omega \wedge \sigma-\omega \wedge \omega \wedge \omega, \\
c^{*}(Z, Z, Z) & =-6 l_{\hat{v}} \hat{\omega} \wedge \hat{\sigma}-\hat{\omega} \wedge \hat{\omega} \wedge \hat{\omega},
\end{aligned}
$$

where the symbol $\iota_{v}$ denotes the contraction by the vector $v$.

We will also need the weighted dual vectors. These are elements of the bundle $N \simeq \operatorname{det} T^{*} M \otimes E^{*}$. In terms of $G L(6)$ tensors, $N$ decomposes as

$$
N \simeq T^{*} M \oplus \Lambda^{4} T^{*} M \oplus\left(T^{*} M \otimes \Lambda^{6} T^{*} M\right),
$$

with sections $Z_{b}=\lambda+\rho+\tau$. The bundle $N$ is obtained from the symmetric product of two generalised vectors via the map $\otimes_{N}: E \otimes E \rightarrow N$ with

$$
\begin{aligned}
& \left.\lambda=v\lrcorner \omega^{\prime}+v^{\prime}\right\lrcorner \omega, \\
& \left.\rho=v\lrcorner \sigma^{\prime}+v^{\prime}\right\lrcorner \sigma-\omega \wedge \omega^{\prime}, \\
& \tau=j \omega \wedge \sigma^{\prime}+j \omega^{\prime} \wedge \sigma .
\end{aligned}
$$

The three-form and six-form gauge potentials of eleven-dimensional supergravity embed in the adjoint bundle adj $F$, which transforms in the $\mathbf{1} \oplus \mathbf{7 8}$ of $E_{6(6)}$. In terms of $G L(6)$ tensors, adj $F$ is defined as

$$
\operatorname{adj} F \simeq \mathbb{R} \oplus\left(T M \otimes T^{*} M\right) \oplus \Lambda^{3} T^{*} M \oplus \Lambda^{6} T^{*} M \oplus \Lambda^{3} T M \oplus \Lambda^{6} T M,
$$

so that its sections are local sums

$$
R=l+r+a+\tilde{a}+\alpha+\tilde{\alpha},
$$

with $l \in \mathbb{R}, r \in \operatorname{End}(T M), a \in \Lambda^{3} T^{*} M$ a three-form, $\tilde{a} \in \Lambda^{6} T^{*} M$ a six-form, $\alpha$ a three vector, and $\tilde{\alpha}$ a six vector. The $\mathfrak{e}_{6(6)}$ Killing form on two elements of the adjoint bundle is given by

$$
\left.\left.\left.\left.\operatorname{tr}\left(R, R^{\prime}\right)=\frac{1}{2}\left(\frac{1}{3} \operatorname{tr}(r) \operatorname{tr}\left(r^{\prime}\right)+\operatorname{tr}\left(r r^{\prime}\right)+\alpha\right\lrcorner a^{\prime}+\alpha^{\prime}\right\lrcorner a-\tilde{\alpha}\right\lrcorner \tilde{a}^{\prime}-\tilde{\alpha}^{\prime}\right\lrcorner \tilde{a}\right) .
$$

The action of an adjoint element $R$ on a generalised vector $V \in \Gamma(E)$ is defined as

$$
\begin{aligned}
v^{\prime} & =l v+r \cdot v+\alpha\lrcorner \omega-\tilde{\alpha}\lrcorner \sigma, \\
V^{\prime}=R \cdot V \quad & \left.\left.\omega^{\prime}=l \omega+r \cdot \omega+v\right\lrcorner a+\alpha\right\lrcorner \sigma, \\
\sigma^{\prime} & =l \sigma+r \cdot \sigma+v\lrcorner \tilde{a}+a \wedge \omega,
\end{aligned}
$$

and on a dual generalised vector $Z$ as

$$
\begin{aligned}
\hat{v}^{\prime} & =-l \hat{v}+r \cdot \hat{v}-\hat{\omega}\lrcorner a+\hat{\sigma}\lrcorner \tilde{a}, \\
Z^{\prime}=R \cdot Z \quad \hat{\omega}^{\prime} & =-l \hat{\omega}+r \cdot \hat{\omega}-\alpha\lrcorner \hat{v}-\hat{\sigma}\lrcorner a, \\
\hat{\sigma}^{\prime} & =-l \hat{\sigma}+r \cdot \hat{\sigma}-\tilde{\alpha}\lrcorner \hat{v}-\alpha \wedge \hat{\omega} .
\end{aligned}
$$


The action of an adjoint element $R$ on another adjoint element $R^{\prime}$ is the commutator, $R^{\prime \prime}=\left[R, R^{\prime}\right]$, which in components reads

$$
\begin{aligned}
& l^{\prime \prime}=\left.\left.\left.\left.\frac{1}{3}(\alpha\lrcorner a^{\prime}-\alpha^{\prime}\right\lrcorner a\right)+\frac{2}{3}\left(\tilde{\alpha}^{\prime}\right\lrcorner \tilde{a}-\tilde{\alpha}\right\lrcorner \tilde{a}^{\prime}\right), \\
& r^{\prime \prime}= {\left.\left.\left.\left.\left[r, r^{\prime}\right]+j \alpha\right\lrcorner j a^{\prime}-j \alpha^{\prime}\right\lrcorner j a-\frac{1}{3}(\alpha\lrcorner a^{\prime}-\alpha^{\prime}\right\lrcorner a\right) \mathbb{I}, } \\
&\left.\left.\left.\left.+j \tilde{\alpha}^{\prime}\right\lrcorner j \tilde{a}-j \tilde{\alpha}\right\lrcorner j \tilde{a}^{\prime}-\frac{2}{3}\left(\tilde{\alpha}^{\prime}\right\lrcorner \tilde{a}-\tilde{\alpha}\right\lrcorner \tilde{a}^{\prime}\right) \mathbb{I}, \\
& a^{\prime \prime}=\left.\left.r \cdot a^{\prime}-r^{\prime} \cdot a+\alpha^{\prime}\right\lrcorner \tilde{a}-\alpha\right\lrcorner \tilde{a}^{\prime}, \\
& \tilde{a}^{\prime \prime}= r \cdot \tilde{a}^{\prime}-r^{\prime} \cdot \tilde{a}-a \wedge a^{\prime}, \\
&\left.\left.\alpha^{\prime \prime}=r \cdot \alpha^{\prime}-r^{\prime} \cdot \alpha+\tilde{\alpha}^{\prime}\right\lrcorner a-\tilde{\alpha}\right\lrcorner a^{\prime}, \\
& \tilde{\alpha}^{\prime \prime}=r \cdot \tilde{\alpha}^{\prime}-r^{\prime} \cdot \tilde{\alpha}-\alpha \wedge \alpha^{\prime},
\end{aligned}
$$

where $\cdot$ denotes the $\mathfrak{g l}(6)$ action.

We conclude this section with the explicit expression of the generalised metric in terms of the supergravity fields. Recall that the generalised metric is a positive-definite, symmetric rank-2 tensor on the generalised tangent bundle,

$$
\begin{aligned}
G: E \otimes E & \rightarrow \mathbb{R}^{+} \\
\quad\left(V, V^{\prime}\right) & \rightarrow G\left(V, V^{\prime}\right)=G_{M N} V^{M} V^{\prime N},
\end{aligned}
$$

where $V$ and $V^{\prime}$ are generalised vectors, and encodes the degrees of freedom of elevendimensional supergravity with components only in the internal manifold. It is more convenient to use the inverse generalised metric. This acts on the dual generalised vectors and in terms of supergravity fields is given by

$$
\begin{aligned}
\left(G^{-1}\right)^{m n} & =e^{2 \Delta} g^{m n} \\
\left(G^{-1}\right)^{m}{ }_{n_{1} n_{2}} & =e^{2 \Delta} g^{m p} A_{p n_{1} n_{2}} \\
\left(G^{-1}\right)^{m} n_{1} \ldots n_{5} & =e^{2 \Delta} g^{m p}\left(A_{p\left[n_{1} n_{2}\right.} A_{\left.n_{3} n_{4} n_{5}\right]}+\tilde{A}_{p n_{1} \ldots n_{5}}\right) \\
\left(G^{-1}\right)_{m_{1} m_{2} n_{1} n_{2}}= & e^{2 \Delta}\left(g_{m_{1} m_{2}, n_{1} n_{2}}+g^{p q} A_{p m_{1} m_{2}} A_{\left.q n_{1} n_{2}\right]}\right) \\
\left(G^{-1}\right)_{m_{1} m_{2} n_{1} \ldots n_{5}}= & e^{2 \Delta}\left[g_{m_{1} m_{2},\left[n_{1} n_{2}\right.} A_{\left.n_{3} n_{4} n_{5}\right]}\right. \\
& \quad+g^{p q}\left(A_{p m_{1} m_{2}}\left(A_{q\left[n_{1} n_{2}\right.} A_{\left.n_{3} n_{4} n_{5}\right]}+\tilde{A}_{q n_{1} \ldots n_{5}}\right)\right] \\
\left(G^{-1}\right)_{m_{1} \ldots m_{5} n_{1} \ldots n_{5}}= & e^{2 \Delta}\left[g_{m_{1} \ldots m_{5}, n_{1} \ldots n_{5}}\right. \\
& \left.\quad+g^{p q}\left(A_{p\left[m_{1} m_{2}\right.} A_{\left.m_{3} m_{4} m_{5}\right]}+\tilde{A}_{p m_{1} \ldots m_{5}}\right)\left(A_{q\left[n_{1} n_{2}\right.} A_{\left.n_{3} n_{4} n_{5}\right]}+\tilde{A}_{q n_{1} \ldots n_{5}}\right)\right],
\end{aligned}
$$

where $\Delta$ is the warp factor, $g$ the internal, $A$ and $\tilde{A}$ the components of the three- and six-form potentials on the internal manifold $M$. Moreover, $g_{m_{1} m_{2}, n_{1} n_{2}}=g_{m_{1}\left[n_{1}\right.} g_{\left.\left|m_{2}\right| n_{2}\right]}$, and similarly for $g_{m_{1} \ldots m_{5}, n_{1} \ldots n_{5}}$.

\subsection{Generalised Structures for $\mathcal{N}=4$ and $\mathcal{N}=2$ Truncations}

The number of supersymmetries of the truncated theory is determined by the number of $G_{S}$ singlets in the generalised spinor bundle $\mathcal{S}$. For compactifications of elevendimensional supergravity to five dimensions, $\mathcal{S}$ transforms in the $\mathbf{8}$ of $U s p(8)$, the double cover of the maximal compact subgroup $U s p(8) / \mathbb{Z}_{2}$ of $E_{6(6)}$.

For maximally supersymmetric truncations, the generalised structure group is the identity, and all higher dimensional supercharges are preserved, giving eight supercharges in the truncated theory. In this case, the R-symmetry group is $U s p(8)$. To construct truncations with reduced supersymmetry, we need to break Usp (8) by considering larger structure groups. For half-maximal truncations, the lower-dimensional R-symmetry is Usp (4), which embeds in Usp (8) as

$$
U s p(8) \supset U s p(4)_{R} \times U s p(4)
$$


so that the largest possible structure group must be $G_{S}=U_{s p}(4)_{S}$. Similarly, for $\mathcal{N}=2$ truncations, the R-symmetry is $S U(2)$, giving as the largest structure group $U s p(6)$.

These two cases lead to minimal five-dimensional supergravities. To have theories with extra matter fields, the structure groups must be broken into smaller ones to allow for extra $G_{S}$ invariant generalised tensors. However, the possibilities are severely constrained by the condition that no extra singlet spinors appear. In what follows, we always consider continuous structure groups, so we do not exclude the existence of other truncations with discrete structure groups.

\section{3. $\mathcal{N}=4$ Truncations}

Half-maximal truncations of eleven-dimensional supergravity to five dimensions correspond to the structure groups

$$
G_{S}=S O(5-n) \quad n=0, \ldots 3 .
$$

The structure $G_{S}$ is defined by a set of $6+n$ invariant generalised vectors, $\mathcal{K}_{0}$ and $\mathcal{K}_{i}$, with $i=1, \ldots, 5+n$ satisfying

$$
\begin{aligned}
& c\left(\mathcal{K}_{0}, \mathcal{K}_{i}, \mathcal{K}_{i}\right)=\eta_{i j} \operatorname{vol}_{6} \quad \\
& c\left(\mathcal{K}_{0}, \mathcal{K}_{0}, V\right)=0, \\
& c\left(\mathcal{K}_{i}, \mathcal{K}_{j}, \mathcal{K}_{k}\right)=0
\end{aligned} \quad \forall V \in \Gamma(E),
$$

where $\eta_{i j}=\operatorname{diag}(-1,-1,-1,-1,-1,+1, \ldots,+1)$ is the flat $S O(5, n)$ metric, $\operatorname{vol}_{6}$ is the volume of the manifold $M$, and $c\left(V, V^{\prime}, V^{\prime \prime}\right)$ is the $E_{6(6)}$ cubic invariant. The invariant vectors $\mathcal{K}_{i}$ are normalised as

$$
\eta\left(\mathcal{K}_{i}, \mathcal{K}_{j}\right)=\eta_{i j}
$$

To see how the $G_{S}$-structure is obtained, recall that the R-symmetry group of halfmaximal supergravities in five dimensions is $U_{s} p(4)_{R}$. This means that $U_{s} p(8)$ must be broken into

$$
U_{s p}(8) \supset U_{s p}(4)_{R} \times U_{s p}(4)_{S}
$$

where the factor $U_{s p}(4)_{R}$ is identified with the R-symmetry, while the other $U_{s} p(4)_{S}$ is the (double cover) of the $G_{S}$-structure group. The spinorial representation of $U s p(8)$ decomposes as

$$
8=(4,1) \oplus(1,4),
$$

and we see that there are indeed four $\tilde{G}_{S}$ singlets that transform in the 4 of $U s p(4)_{R}$ and can therefore be identified with the supersymmetry parameters of half-maximal supergravity.

The structure group $U s p(4)_{S} \sim S O(5)$ embeds in $E_{6(6)}$ as

$$
E_{6(6)} \supset S O(1,1) \times S O(5) \times S O(5) .
$$

The decomposition of the generalised bundle under (55)

$$
27=(5,1) \oplus(4,4) \oplus(\mathbf{1}, 5) \oplus(\mathbf{1}, \mathbf{1})
$$

contains six invariant generalised vectors $\mathcal{K}_{0}$ and $\mathcal{K}_{i}, i=1, \ldots, 5$. The decomposition of the adjoint bundle

$$
\mathbf{7 8}=(\mathbf{1 0}, \mathbf{1})_{0} \oplus(\mathbf{5}, \mathbf{5})_{0} \oplus(\mathbf{1}, \mathbf{1 0})_{0} \oplus(\mathbf{4}, \mathbf{4})_{-3} \oplus(\mathbf{4}, \mathbf{4})_{3} \oplus(\mathbf{1}, \mathbf{1})_{0}
$$

gives ten plus one singlets corresponding to the generators of the commutant $G=$ $S O(1,1) \times S O(5)$ of the structure group in $E_{6(6)}$. Notice, however, that these adjoint singlets can be constructed as tensor products of the invariant vectors and their duals, $\mathcal{K}^{*} \times{ }_{\text {adj }} \mathcal{K}$, and therefore do not play a role in the definition of the structure group. The $G_{S}$-structure is completely determined by the generalised vectors. 
According to the dictionary of the previous section, the six invariant generalised vectors $\mathcal{K}_{0}$ and $\mathcal{K}_{i}$ give six vectors in the truncated theory, while the scalar manifold is (see (18))

$$
\mathcal{M}=\frac{\operatorname{Com}_{E_{6(6)}}\left(S O(5)_{S}\right)}{\operatorname{Com}_{U s p(8)}\left(S O(5)_{S}\right)}=S O(1,1)
$$

so that there is only one scalar. This is the field content of the gravity multiplet of halfmaximal supergravity in five dimensions. Thus, a $G_{S}=S O(5)$ gives a truncation to pure supergravity.

To have an extra matter field, we need to further break the structure group in such a way that there are no extra singlets in the decomposition of the spinor representation of $U s p(8)$. This requirement restricts the possible structure groups to the following subgroups of $S O(5)_{S}$ :

$$
G_{S}=S O(5-n) \quad G_{S}=S U(2) \times U(1) \quad G_{S}=U(1) \times U(1),
$$

with $n=0, \ldots, 3$. It is easy to verify that the last two groups above have the same commutant in $E_{6(6)}$ and the same $G_{S}$-singlets as the case of $G_{S}=S O(5-n)$ with $n=1$. This means that they give rise to the same truncations as $G_{S}=S O(4)$, and we can then focus on the family of $G_{S}=S O(5-n)$ structures.

From the embedding $E_{6(6)} \supset S O(1,1) \times S O(5, n) \times S O(5-n)$, the generalised tangent bundle decomposes as

$$
27=(\mathbf{5}+\mathbf{n}, \mathbf{1})_{2} \oplus(\mathbf{1}, \mathbf{5}-\mathbf{n})_{2} \oplus(\mathbf{4}, \mathbf{4})_{-1} \oplus(\mathbf{1}, \mathbf{1})_{-4},
$$

where the subscripts denote the $S O(1,1)$ weights. Thus, we obtain $6+n$ singlets transforming in the $\mathbf{1}_{-4} \oplus(\mathbf{5}+\mathbf{n})_{2}$ of the commutant $\mathcal{G}=O(1,1) \times S O(5, n)$ :

$$
\left\{\mathcal{K}_{I}\right\}=\left\{\mathcal{K}_{0}, \mathcal{K}_{i}\right\}, \quad I=0,1, \ldots, 5+n .
$$

The $\mathcal{K}_{I}$ are in one-to-one correspondence with the vectors of the half-maximal supergravity: six of them come from the gravity multiplet, and $n$ from the additional vector multiplets.

The scalars of the truncated theory now parameterise the coset

$$
\mathcal{M}_{\text {scal }}=O(1,1) \times \frac{S O(5, n)}{S O(5) \times S O(n)},
$$

which matches the standard structure of the scalar manifold for half-maximal supergravity coupled to $n$ vector multiplets [46]. The single scalar in the gravity multiplet parameterises the $O(1,1)$ factor $^{6}$, while the scalars in the vector multiplets parameterise the $\frac{S O(5, n)}{S O(5) \times S O(n)}$ coset space.

The two-form fields of the truncated theory are determined by the $S O(5-n)$ singlets in the bundle $N$. In the decomposition under $S O(1,1) \times S O(5, n) \times S O(5-n) \subset E_{6(6)}$, we find again $6+n$ singlets, $Z_{b}^{0}, Z_{b}^{i}$ with $i=1, \ldots, 5+n$. It is natural to normalise them via the pairing (37)

$$
\left\langle Z_{b}^{I}, \mathcal{K}_{J}\right\rangle=\delta^{I}{ }_{J} \operatorname{vol}_{6}
$$

Let us consider now the gauging of the truncated theory. This is determined by the intrinsic torsion of the structure, which encodes the embedding tensor of the reduced theory. Since in this case, the $G_{S}$-structure is defined by generalised vectors only, all the information about the intrinsic torsion is encoded in (101):

$$
L_{\mathcal{K}_{I}} \mathcal{K}_{J}=X_{I J}{ }^{K} \mathcal{K}_{K},
$$

where the matrices $\left(X_{I}\right)_{J}{ }^{K}=X_{I J}{ }^{K}$ are the generators of the algebra 


$$
\left[X_{I}, X_{J}\right]=-X_{I J}{ }^{K} X_{K}
$$

The gaugings of half-maximal supergravity in five dimensions have been analysed in [46]. The embedding tensor has components

$$
f_{i j k}=f_{i j k]}, \quad \xi_{i j}=\xi_{[i j]}, \quad \xi_{i},
$$

satisfying

$$
3 f_{[i j}{ }^{k} f_{l m] k}=2 f_{[l m i} \xi_{j]}, \quad \xi_{i}^{m} f_{m j k}=\xi_{i} \xi_{j k}-\xi_{[j} \xi_{k] i},
$$

and

$$
3 f_{i j k} \xi^{k}=0, \quad \xi_{i j} \xi^{j}=0, \quad \xi_{i} \xi^{i}=0,
$$

where the indices are raised/lowered using the $S O(5, n)$ metric $\eta_{i j}$.

The components of the embedding tensor are identified with the components of the gauge group generators $\left(X_{I}\right)_{J}{ }^{K}$ in (65). Using the composite index $I=\{0, i\}$, we can assemble (66) as

$$
X_{i j}{ }^{k}=-f_{i j}{ }^{k}, \quad X_{0 i}{ }^{j}=-\xi_{i}{ }^{j}, \quad X_{0 i}{ }^{0}=-\xi_{i} .
$$

Note that in generalised geometry, the algebraic conditions $f_{A B C}=f_{[A B C]}$ and $\xi_{A B}=$ $\xi_{[A B]}$ follow from consistency of the generalised algebra (64) with the conditions (51) and (52).

The $G_{S}=S O(5-n)$ structure completely determines the number $n$ of vector multiplets and the embedding tensor from the generalised $S O(5-n)$ structure and therefore fully characterises the five-dimensional half-maximal supergravity theory that is obtained after truncation. To complete the truncation procedure, we need to discuss how the lowerdimensional fields embed into the higher-dimensional ones. The general ideas about the truncation ansatz were discussed in Section 3 . Here, we will specify them to the truncations of eleven-dimensional supergravity to half-maximal supergravities in five dimensions.

We can organise the eleven-dimensional supergravity fields into $E_{6(6)}$ representations as in Section 3. The fields with only legs on $M$ are organised into the inverse generalised metric

$$
G^{M N}(x, y) \leftrightarrow\left\{\Delta, g_{m n}, A_{m_{1} m_{2} m_{3}}, \tilde{A}_{m_{1} \ldots m_{6}}\right\},
$$

those with one external leg into generalised vectors

$$
\mathcal{A}_{\mu}{ }^{M}(x, y)=\left\{h_{\mu}{ }^{m}, A_{\mu m n}, \tilde{A}_{\mu m_{1} \ldots m_{5}}\right\},
$$

and those with two external legs into weighted dual vectors

$$
\mathcal{B}_{\mu v M N}(x, y)=\left\{A_{\mu \nu m}, \tilde{A}_{\mu v m_{1} \ldots m_{4}}, \tilde{g}_{\mu \nu m_{1} \ldots m_{6}, n}\right\},
$$

where we will not need the last term, related to the dual graviton. In all the above expressions, $M, N$ label indices in the $\mathbf{2 7}$ or $\overline{\mathbf{2 7}}$.

Then, the bosonic truncation ansatz is obtained by expanding these fields on the $G_{S}$ invariant tensors. For the vector fields and the two-forms, we have

$$
\begin{aligned}
& \mathcal{A}_{\mu}^{M}(x, y)=\sum_{\mathcal{I}=0}^{5+n} \mathcal{A}_{\mu}^{I}(x) \mathcal{K}_{I}^{M}(y), \\
& \mathcal{B}_{\mu v M N}(x, y)=\sum_{I=0}^{5+n} \mathcal{B}_{\mu v I}(x) Z^{I}{ }_{b M N}(y),
\end{aligned}
$$

where $\mathcal{A}_{\mu}^{I}$ and $\mathcal{B}_{\mu v I}$ are the five-dimensional supergravity vector fields and two-forms, respectively. 
The ansatz for the scalar fields is more involved as it requires the explicit expression for the generalised metric $G^{M N}$ in terms of the $G_{S}$ invariant tensors $\mathcal{K}_{I}$ [23]. The inverse metric acting on two dual generalised vectors is

$$
G^{-1}(Z, Z)=G_{0}^{-1}(Z, Z)+G_{10}^{-1}(Z, Z)+G_{16}^{-1}(Z, Z),
$$

where the subscripts denote $S O(5,5)$ representations ${ }^{7}$ and

$$
\begin{aligned}
& G_{0}^{-1}(Z, Z)=\left\langle Z, \mathcal{K}_{0}\right\rangle\left\langle Z, \mathcal{K}_{0}\right\rangle \\
& G_{10}^{-1}(Z, Z)=2 \delta^{i j}\left\langle Z, \mathcal{K}_{i}\right\rangle\left\langle Z, \mathcal{K}_{j}\right\rangle+\eta^{-1}(Z, Z) \\
& G_{16}^{-1}(Z, Z)=-4 \sqrt{2}\left\langle Z, \mathcal{K}_{1} \cdots \mathcal{K}_{5} \cdot Z\right\rangle
\end{aligned}
$$

with $\eta^{-1}(Z, Z)$ being the inverse of the $S O(5,5)$ metric.

The generalised metric entering the scalar ansatz is then constructed by plugging into the expressions above the "dressed" invariant vectors, which are obtained by multiplying the $\mathcal{K}_{I}$ with a representative of the scalar coset (62)

$$
\tilde{\mathcal{K}}_{0}=\Sigma^{2} K_{0}, \quad \tilde{\mathcal{K}}_{a}=\Sigma^{-1} \mathcal{V}_{a}{ }^{i} \mathcal{K}_{i}, \quad \tilde{\mathcal{K}}_{\underline{a}}=\Sigma^{-1} \mathcal{V}_{\underline{a}}{ }^{i} \mathcal{K}_{i}
$$

Here, $\Sigma$ is a scalar parameterising the $O(1,1)$ factor in $(62)$, while $\left(\mathcal{V}_{a}{ }^{i}, \mathcal{V}_{\underline{a}}{ }^{i}\right)^{T} \in S O(5, n)$ is the inverse of the coset representative of $\frac{S O(5, n)}{S O(5) \times S O(n)}$, with $a=1, \ldots, 5$ and $\underline{a}=1, \ldots, n$ local $S O(5)$ and $S O(n)$ indices, respectively.

The expression for the inverse generalised metric is then

$$
\begin{aligned}
G^{-1}(Z, Z) & =G_{0}^{-1}(Z, Z)+G_{10}^{-1}(Z, Z)+G_{10}^{-1}(Z, Z) \\
& =\Sigma^{4}\left\langle Z, K_{0}\right\rangle\left\langle Z, K_{0}\right\rangle+\Sigma^{-2}\left(2 \delta^{a b} \mathcal{V}_{a}{ }^{i} \mathcal{V}_{b}{ }^{j}\left\langle Z, K_{i}\right\rangle\left\langle Z, K_{j}\right\rangle+\eta^{-1}(Z, Z)\right) \\
& -\frac{4 \sqrt{2}}{5 !} \Sigma \epsilon^{a b c d e} \mathcal{V}_{a}{ }^{i} \mathcal{V}_{b}{ }^{j} \mathcal{V}_{c}{ }^{k} \mathcal{V}_{d}{ }^{l} \mathcal{V}_{e}{ }^{m}\left\langle Z, K_{i} \cdots K_{m} \cdot Z\right\rangle
\end{aligned}
$$

and the scalar ansatz is obtained by equating (76) with the expression (48), which encodes all supergravity fields with purely internal indices. By separating the different tensorial structures on the internal manifold $M$, we obtain the scalar ansatz for the individual higher-dimensional supergravity fields ${ }^{8}$.

\section{4. $\mathcal{N}=2$ Truncations}

In $\mathcal{N}=2$ supergravity in five dimensions, the R-symmetry group is $S U(2)$. This embeds in $E_{6(6)}$ as

$$
U s p(8) \supset S U(2)_{R} \times U s p(6),
$$

where the factor $U s p(6)$ corresponds to the structure group. Under the embedding (77), the spinorial representation of $U s p(8)$ decomposes as

$$
8=(6,1) \oplus(1,2)
$$

where the two $U s p(6)$ singlets give the $S U(2)_{R}$ doublet of supersymmetry parameters of $\mathcal{N}=2$ supersymmetry.

Decomposing the $\mathbf{2 7}$ and $\mathbf{7 8}$ representations of $E_{6(6)}$ under (77), we find one singlet generalised vector $K$ of positive norm with respect to the $E_{6(6)}$ cubic invariant,

$$
c(K, K, K)=6 \kappa^{2}>0,
$$

where $\kappa$ is a section of $\left(\operatorname{det} T^{*} M\right)^{1 / 2}$, and an $S U(2)$ triplet of adjoint tensors $J_{\alpha} \in \Gamma(\operatorname{adj} F)$, with $\alpha=1,2,3$, satisfying

$$
\left[J_{\alpha}, J_{\beta}\right]=2 \epsilon_{\alpha \beta \gamma} J_{\gamma}, \quad \operatorname{Tr}\left(J_{\alpha} J_{\beta}\right)=-\delta_{\alpha \beta} .
$$


The globally defined vector $K \in \Gamma(E)$ with positive norm is called a vector-multiplet structure, or $\mathrm{V}$ structure. A triplet of $J_{\alpha} \in \Gamma(\operatorname{adj} F)$ that defines the highest root $\mathfrak{s u}_{2}$ subalgebra of $\mathfrak{e}_{6(6)}$ and satisfies the conditions (80) is called a hypermultiplet structure, or $\mathrm{H}$ structure. Together, when they satisfy the compatibility conditions

$$
J_{\alpha} \cdot K=0, \quad c(K, K, K)=\frac{1}{6} \kappa^{2} \operatorname{Tr}\left(J_{\alpha} J_{\beta}\right) .
$$

$K$ and $J_{\alpha}$ define an HV structure or $U s p(6)$ structure.

An HV structure corresponds to truncations to minimal $\mathcal{N}=2$ supergravity in five dimensions. Indeed, the vector $K$ is in one-to-one correspondence with the five-dimensional graviphoton, while the three Js in the adjoint give the generators of the $S U(2)_{R} R$-symmetry. This is also confirmed by looking at the scalar manifold, which from (18) is trivial:

$$
\mathcal{M}=\frac{\operatorname{Com}_{E_{6(6)}}(U s p(6))}{\operatorname{Com}_{U s p(8)}(U s p(6))}=\mathbb{R}^{+}
$$

as $\operatorname{Com}_{E_{6(6)}}(U s p(6))=\operatorname{Com}_{U s p(8)}(U s p(6))=S U(2)$.

To have extra matter multiplets, as for the half-maximal case, the structure group must be reduced to a $G_{S} \subset U_{s p}(6)$ in order to have an extra singlet in the $\mathbf{2 7}$ and $\mathbf{7 8}$. As before, the allowed breakings are restricted by the condition that there are no new singlets in the decomposition of the spinorial representation of $U s p(8)$.

Thus, a generic $G_{S} \subset U s p(6)$ corresponding to $\mathcal{N}=2$ supersymmetry in five dimensions is defined by the set

$$
\left\{K_{I}, J_{A}\right\}
$$

of $G_{S}$-invariant independent generalised vectors

$$
K_{I}, \quad I=0, \ldots, n_{V},
$$

and $G_{S}$-invariant elements of the 78

$$
J_{A}, \quad A=1, \ldots, \operatorname{dim} \mathcal{H},
$$

that also satisfy the condition

$$
J_{A} \cdot K_{I}=0 \quad \forall I \text { and } \forall A .
$$

Note that a priori, there can be other singlets in the adjoint bundle that do not satisfy (86). These are given by $K_{I} \times_{\text {adj }} K_{J}^{*}$, where $K_{J}^{*}$ is the dual of the generalised vector $K_{J}$, and $\times_{\text {adj }}$ is the projection onto the adjoint bundle. These extra singlets generate the isometries of the vector scalar manifold in five dimensions, while the $J_{A}$ generate the isometry group of the hyper-multiplet scalar manifold $\mathcal{H} \subset \operatorname{Com}_{E_{6(6)}}\left(G_{S}\right)$, so that

$$
\left[J_{A}, J_{B}\right]=f_{A B}{ }^{C} J_{C},
$$

with $f_{A B}{ }^{C}$ being the structure constants of $\mathcal{H}$.

We can always normalise the $K_{I}$ to satisfy

$$
c\left(K_{I}, K_{J}, K_{K}\right)=6 \kappa^{2} C_{I J K},
$$

with $C_{I J K}$ a symmetric, constant tensor, and normalise the adjoint singlets to

$$
\operatorname{Tr}\left(J_{A} J_{B}\right)=\eta_{A B},
$$

where $\eta_{A B}$ is a diagonal matrix with -1 and +1 entries in correspondence with compact and non-compact generators of $\mathcal{H}$, respectively. 
The generalised metric is given in terms of the $G_{S}$ invariant tensors as [24]

$$
G(V, V)=3\left(3 \frac{c(K, K, V)^{2}}{c(K, K, K)^{2}}-2 \frac{c(K, V, V)}{c(K, K, K)}+4 \frac{c\left(K, J_{3} \cdot V, J_{3} \cdot V\right)}{c(K, K, K)}\right) .
$$

The $G_{S}$-structure determines the field content of the truncated theory. The singlet generalised vectors are in one-to-one correspondence with the vector of the truncated theory, namely the graviphoton and the vectors in the vector multiplets. The scalar manifold is given by

$$
\mathcal{M}=\frac{\operatorname{Com}_{E_{6(6)}}\left(G_{S}\right)}{\operatorname{Com}_{U s p(8) / \mathbb{Z}_{2}}\left(G_{S}\right)} .
$$

The expression above can be interpreted in the following way. Given the $G_{S} \subset U_{s p}(6)$ structure, one defines a $U_{s p}(6)$ structure where the $K$ and $J_{\alpha}$ are combinations of the $K_{I}$ and $J_{A}$ and then builds a generalised metric as in (90). Clearly, there are many ways to define such a $U_{s p}(6)$ structure, depending on the way $K$ and $J_{\alpha}$ are expressed in terms of $K_{I}$ and $J_{A}$. The parameterisation of $K$ and $J_{\alpha}$ in terms of $K_{I}$ and $J_{A}$ provides a set of deformations of a reference $U s p(6)$-invariant metric that corresponds to acting on the structure with elements of $E_{6(6)}$ that commute with $G_{S}$, modulo elements of $U s p(8) / \mathbb{Z}_{2}$ that commute with $G_{S}$, thus giving (91).

The requirement (86) implies that, as expected from $\mathcal{N}=2$ supergravity, the space $\mathcal{M}$ splits in the product

$$
\mathcal{M}=\mathcal{M}_{\mathrm{V}} \times \mathcal{M}_{\mathrm{H}}
$$

of the $\mathrm{V}$ structure moduli space $\mathcal{M}_{\mathrm{V}}$ and the $\mathrm{H}$ structure one, $\mathcal{M}_{\mathrm{H}}$. Notice that by construction, these are always symmetric spaces.

The vector moduli space $\mathcal{M}_{\mathrm{V}}$ corresponds to deformations of $K$ that leave $J_{\alpha}$ invariant and is obtained by expressing the generalised vector $K$ as a linear combination of the invariant vectors $K_{I}$ :

$$
K=h^{I} K_{I}, \quad I=0, \ldots, n_{\mathrm{V}},
$$

where $h^{I}$ are $n_{\mathrm{V}}+1$ real scalars. Because of the condition (79), the parameters $h^{I}$ must satisfy

$$
C_{I J K} h^{I} h^{J} h^{K}=1,
$$

and therefore define an $n_{\mathrm{V}}$-dimensional hypersurface,

$$
\mathcal{M}_{\mathrm{V}}=\left\{h^{I}: C_{I J K} h^{I} h^{J} h^{K}=1\right\} .
$$

This is the V structure moduli space, which gives the vector multiplet scalar manifold in five-dimensional supergravity. Using the generalised metric, we can also derive the metric on $\mathcal{M}_{\mathrm{V}}$ as

$$
a_{I J}=\frac{1}{3} G\left(K_{I}, K_{J}\right) .
$$

The $\mathrm{H}$ structure moduli space describes deformations of $J_{\alpha}$ that leave $K$ invariant and corresponds to the space of choices of highest root $\mathfrak{s u}(2)$ algebrae in the algebra spanned by the $J_{A}$. Concretely, we can start from a reference $\mathfrak{j} \simeq \mathfrak{s u}(2)$ algebra and then act on a basis $j_{a}$ of $\mathfrak{j}$ by the group elements $h \in \mathcal{H}$ :

$$
J_{\alpha}=\operatorname{adj}_{\mathcal{H}} j_{\alpha}=h j_{\alpha} h^{-1} .
$$

Clearly, $h \in S U(2)_{R}=\exp j$ and $h \in \operatorname{Com}_{\mathcal{H}}\left(S U(2)_{R}\right)$ act trivially on the $j_{a}$ and have to be modded out. We obtain the coset

$$
\mathcal{M}_{\mathrm{H}}=\frac{\mathcal{H}}{S U(2)_{R} \times \operatorname{Com}_{\mathcal{H}}\left(\operatorname{SU}(2)_{R}\right)} .
$$


where $\mathcal{M}_{\mathrm{H}}$ are "Wolf spaces" and are all quaternionic-Kähler, as expected from the hyperscalar manifold in five-dimensional supergravity.

By analysing the possible breakings of $U s p(6)$ that only give two singlets in the decomposition of the spinorial representation of $U_{s p}(8)$, it is possible to classify all allowed truncations of eleven-dimensional supergravity to five dimensions that are truly $\mathcal{N}=2$ supersymmetric [44]. In Table 2 below, we list all the possible truncations that correspond to a continuous $G_{S} \subseteq U s p(6)$ structure group.

Table 2. Allowed $\mathcal{N}=2$ truncations of 11- $d$ supergravity.

\begin{tabular}{|c|c|c|c|}
\hline$n_{\mathrm{V}} \quad n_{\mathrm{H}}$ & 0 & 1 & 2 \\
\hline 0 & $\begin{array}{c}G_{S}=U s p(6) \\
\mathcal{M}=1\end{array}$ & $\begin{array}{c}G_{S}=\operatorname{SU}(3) \\
\mathcal{M}=\frac{\operatorname{SU}(2,1)}{\operatorname{SU}(2) \times U(1)}\end{array}$ & $\begin{aligned} G_{S} & =S O(3) \\
\mathcal{M} & =\frac{G_{2(2)}}{S O(4)}\end{aligned}$ \\
\hline 1 & $\begin{array}{c}G_{S}=S U(2) \times S O(5) \\
\mathcal{M}=\mathbb{R}_{+}\end{array}$ & $\begin{array}{c}G_{S}=S U(2) \times U(1) \\
\mathcal{M}=\mathbb{R}_{+} \times \frac{S U(2,1)}{\operatorname{SU}(2) \times U(1)}\end{array}$ & - \\
\hline 2 & $\begin{array}{l}G_{S}=S U(2) \times S O(4) \\
\mathcal{M}=\mathbb{R}_{+} \times S O(1,1)\end{array}$ & - & - \\
\hline 3 & $\begin{array}{c}G_{S}=S U(2) \times S O(3) \\
\mathcal{M}=\mathbb{R}_{+} \times \frac{S O(2,1)}{S O(2)}\end{array}$ & - & - \\
\hline 4 & $\begin{array}{c}G_{S}=S U(2) \times S O(2) \\
\mathcal{M}=\mathbb{R}_{+} \times \frac{S O(3,1)}{S O(3)}\end{array}$ & $\begin{array}{c}G_{S}=U(1) \\
\mathcal{M}= \\
\mathbb{R}_{+} \times \frac{S O(3,1)}{S O(3)} \times \frac{S U(2,1)}{S U(2) \times U(1)}\end{array}$ & - \\
\hline 5 & $\begin{array}{c}G_{S}=S U(2) \\
\mathcal{M}=\frac{S L(3)}{S O(3)} \\
G_{S}=S U(2) \times \mathbb{Z}_{2} \\
\mathcal{M}=\mathbb{R}_{+} \times \frac{S O(4,1)}{S O 4}\end{array}$ & - & - \\
\hline 6 & $\begin{array}{l}G_{S}=S U(2) \times \mathbb{Z}_{2} \\
\mathcal{M}=\mathbb{R}_{+} \times \frac{S O(5,1)}{S O 5}\end{array}$ & - & - \\
\hline 8 & $\begin{array}{c}G_{S}=U(1) \\
\mathcal{M}=\frac{S L(3, \mathbb{C})}{S U(3)}\end{array}$ & - & - \\
\hline 14 & $\begin{array}{c}G_{S}=\mathbb{Z}_{2} \\
\mathcal{M}=\frac{\operatorname{SU}^{*}(6)}{U s p(6)}\end{array}$ & - & - \\
\hline
\end{tabular}

In Table 2, we recover the fields content of some well-known truncations with only vectors or only hypermultiplets $[47,48]$. Surprisingly, we find a very limited number of truncations with both vectors and hypermultiplets.

Let us stress that the list above is not a list of actual consistent truncations. These are the truncations that are a priori allowed from an algebraic point of view, namely the list of $G_{S} \supseteq U_{s p}(6)$ that have the right features to give $\mathcal{N}=2$ truncations. Here, we assumed that the intrinsic torsion only contains singlet representations of $G_{S}$. Verifying this condition implies analysing the differential properties of the structure, and for this, we need the explicit knowledge of the compactification manifold.

Under the assumption that the $G_{S}$-structure has only singlet intrinsic torsion, we can give the details of how this is related to the embedding tensor of the truncated theory. For $\mathcal{N}=2$ supersymmetry, the embedding tensor splits into two parts [49,50],

$$
\left(\Theta_{\tilde{I}}^{a}, \Theta_{\tilde{I}}^{A}\right),
$$

with $a=1, \ldots, \operatorname{dim} \mathfrak{g}_{\mathrm{V}}$ and $A=1, \ldots, \operatorname{dim} \mathfrak{g}_{\mathrm{H}}$, reflecting the split of the isometry algebra

$$
\mathfrak{g}=\mathfrak{g}_{\mathrm{V}} \oplus \mathfrak{g}_{\mathrm{H}}
$$


into the Lie algebrae of isometries of the vector and hypermultiplet moduli spaces, respectively.

For $\mathcal{N}=2$ truncations, the intrinsic torsion (101) is given by the action of the generalised Lie derivative along the invariant vectors $K_{I}$ on the $G_{S}$-invariant tensor $K_{I}$ and $J_{A}$ :

$$
\begin{aligned}
& L_{K_{I}} K_{J}=\Theta_{I} \cdot K_{J}=\Theta_{I}^{a}\left(t_{a}\right)_{J}{ }^{L} K_{L}:=f_{I J}{ }^{L} K_{L}, \\
& L_{K_{I}} J_{A}=\Theta_{I} \cdot J_{A}=\left[J_{\left(K_{I}\right)} J_{A}\right]=\Theta_{I}^{B} f_{B A}{ }^{C} J_{C}:=p_{I A}{ }^{B} J_{B} .
\end{aligned}
$$

Here, $\left(t_{a}\right)_{J}{ }^{L}$ are the representations of the generators of Lie $\mathcal{G}$ acting on $\mathcal{V}, J_{\left(K_{I}\right)}:=$ $\Theta_{I}^{A} J_{A}$ is an element of the adjoint, and $f_{[I J]}{ }^{L}$ are the structure constants of the gauge algebra, while $f_{A B}{ }^{C}$ are the structure constants of the algebra $H$ acting on the hypers.

Demanding that the $G_{S}$-structure has only singlet intrinsic torsion implies that $f_{I J} K$ and $p_{I A}{ }^{B}$ in (101) are indeed constants and that the equation

$$
\int_{M} \kappa^{2} \operatorname{Tr}\left(J_{A}\left(L_{W} J_{B}\right)\right)=0,
$$

where the generalised vector $W$ satisfies $c\left(K_{I}, K_{J}, W\right)=0$, is satisfied.

The last ingredient for a truncation is again the truncation ansatz. The logic is the same as for half-maximal truncations. The embedding to the vectors of the truncated theory $\mathcal{A}_{\mu}{ }^{I}(x)$ in the higher-dimensional fields is determined by equating (71) to the expansion

$$
\mathcal{A}_{\mu}(x, y)=\mathcal{A}_{\mu}{ }^{I}(x) K_{I}(y) \quad I=0,1, \ldots, n_{\mathrm{V}} .
$$

Similarly, equating (72) to the expansion

$$
\mathcal{B}_{\mu v}(x, y)=\mathcal{B}_{\mu \nu I}(x) K_{b}^{I}
$$

gives the embedding of the two-forms of the truncated theory.

Finally, the scalars are obtained by first defining the $K$ and $J_{\alpha}$ parameterising a family of HV structures

$$
\begin{aligned}
& K(x, y)=h^{I}(x) K_{I}, \\
& J_{\alpha}(x, y)=L(x) j_{\alpha} L(x)^{-1}
\end{aligned}
$$

where $L$ is the representative of the $\operatorname{coset} \mathcal{M}_{\mathrm{H}}$, and then plugging them into the generalised metric (90). Comparing the expression for the generalised metric with its general form (48), we obtain the truncation ansatz for the supergravity fields with only internal indices.

\section{Conclusions}

In this article, we reviewed the applications of exceptional generalised geometry to the study of consistent truncations. In this approach, a central role is played by the notion of the $G$-structure, namely the existence of nowhere-vanishing $G_{S}$-invariant tensors on the internal manifold $M$. We showed that in order to have a consistent truncation of a given supergravity theory on a manifold $M$, this must admit a generalised $G_{S}$-structure with singlet intrinsic torsion. The $G_{S}$ structure completely determines the field content, the amount of supersymmetry, and the gauging of the truncated theory,

Then, this approach provides a systematic way to study consistent truncations in various dimensions and with different amounts of supersymmetry.

As a first example, we briefly recalled how the notion of the $G_{S}$-structure allows one to understand all maximally supersymmetric truncations as generalised Scherck-Schwarz reductions on generalised parallelisable manifolds.

Then, we focussed on eleven-dimensional supergravity, and we studied in detail the truncations to $\mathcal{N}=4$ and $\mathcal{N}=2$ five-dimensional supergravity. In this case, the Eexceptional generalised geometry is based on the $E_{6(6)}$ exceptional group, and we showed how, based on the properties of the $G_{S}$-structure, it is possible to explicitly determine 
the scalar moduli spaces and the embedding tensor of the truncated theories. The same analysis holds for truncations of type IIB supergravity to five dimensions. This means that under the assumption that the $G_{S}$-structure has singlet intrinsic torsion, it possible to determine which five-dimensional supergravity theory can in principle be obtained from the M-theory or type IIB.

It is important to stress that this analysis is not enough to guarantee that the consistent truncation actually exists. To do so, we should be able to explicitly construct manifolds that realise such $G_{S}$ structures with singlet intrinsic torsion. A very interesting direction to explore is to see whether one could derive the differential conditions, such as those derived for generalised Scherck-Schwarz reductions [11], that a manifold should satisfy in order to have a given $G_{S}$-structure with singlet intrinsic torsion.

As this approach has already, and can in the future, give new examples of consistent truncations, it would be nice to study solutions of these theories, such as black holes, black strings, and domain walls and their relevance for the AdS/CFT correspondence.

It would also be interesting to continue the programme of scanning through dimensions and amounts of supersymmetry to have a full classification of the supergravity theories that can be obtained from string or M-theory.

Finally, another direction to explore is how to include the open string sector in the truncations, as this can have interesting applications to fuzz-ball constructions and AdS/CFT.

Funding: This research received no external funding.

Institutional Review Board Statement: Not applicable.

Informed Consent Statement: Not applicable.

Data Availability Statement: Not applicable.

Acknowledgments: I would like to thank all my collaborators on the various articles that are the basis for this review article: Davide Cassani, Oscar De Felice, Gregoire Josse, Emanuel Malek, Charles Strickland-Constable, and Daniel Waldram. I also would like to thank Norma Sanchez for the invitation to contribute to the Open Access Special Issue "Women Physicists in Astrophysics, Cosmology and Particle Physics" to be published in Universe (ISSN 2218-1997, IF 1.752).

Conflicts of Interest: The authors declare no conflict of interest

\section{Notes}

Formally a $G_{S}$-structure defines a $G_{S}$-principal sub-bundle $P$ of the $G L(d, \mathbb{R})$ frame bundle. In most cases, the two definitions are equivalent.

2 We consider only orientable manifolds. Then, $\operatorname{det} T^{*} M$ is trivial and we can define arbitrary powers $\left(\operatorname{det} T^{*} M\right)^{p}$ for any real $p$.

3 A generalised connection $\tilde{D}$ is compatible with the $G_{S}$-structure if $\tilde{D} Q_{i}=0$ for all $Q_{i}$. The definition of a generalised connection is the same as in conventional differential geometry. However, in generalised geometry, the conditions of being torsion free and metric compatible do not uniquely determine the connection. However, only certain projections of the action of the connection appear in the supergravity, and these are unique [35].

4 We do not consider higher form-field degrees of freedom, as in the tensor hierarchy [38,39], since they are dual to the scalar, vector, and two-forms and therefore do not introduce new degrees of freedom. In particular, this means that for $D=4, \mathcal{A}_{\mu}{ }^{M}$ contain both the vectors and their duals, and in $D=6, \mathcal{B}_{\mu \nu}{ }^{M N}$ contain both the two-forms and their duals.

5 One can show [6] that a necessary condition for the existence of a generalised parallelisation satisfying (27) is that $M$ is a coset manifold.

We renamed the $\mathbb{R}^{+} O(1,1)$ to match the standard supergravity literature.

The generalised vector $\mathcal{K}_{0}$ defines an $S O(, 5,5)$ structure that embeds in $E_{6(6)}$ as $E_{6(6)} \supset S O(5,5) \times O(1,1)$.

Note that the coset representative $\left(\mathcal{V}_{i}{ }^{a}, \mathcal{V}_{i}{ }^{a}\right)$ satisfies

$$
\eta_{i j}=-\delta_{a b} \mathcal{V}_{i}^{a} \mathcal{V}_{j}^{b}+\delta_{\underline{a} b} \mathcal{V}_{i}^{\underline{a}} \mathcal{V}^{\underline{b}} \quad M_{i j}=\delta_{a b} \mathcal{V}_{i}^{a} \mathcal{V}_{j}^{b}+\delta_{\underline{a} b} \mathcal{V}_{i}{ }^{\underline{a}} \mathcal{V}^{\underline{b}}
$$

Then, one can define the $S O(5) \times S O(n)$ invariant matrices $2 \delta^{a b} \mathcal{V}_{a}{ }^{A} \mathcal{V}_{b}{ }^{j}=M^{i j}-\eta^{i j}, M^{i j k l m}=\epsilon^{a b c d e} \mathcal{V}_{a}{ }^{i} \mathcal{V}_{b}{ }^{j} \mathcal{V}_{c}{ }^{k} \mathcal{V}_{d}{ }^{l} \mathcal{V}_{e}{ }^{m}$, which appear in half-maximal supergravity in five dimensions [46]. 


\section{References}

1. Duff, M.J.; Nilsson, B.E.; Pope, C.N.; Warner, N.P. On the Consistency of the Kaluza-Klein Ansatz. Phys. Lett. 1984, 149, 90-94. [CrossRef]

2. Cvetic, M.; Lu, H.; Pope, C.N. Consistent Kaluza-Klein sphere reductions. Phys. Rev. 2000, D62, 064028. [CrossRef]

3. Scherk, J.; Schwarz, J.J. How to Get Masses from Extra Dimensions. Nucl. Phys. 1979, 153, 61-88. [CrossRef]

4. De Wit, B.; Nicolai, H. The Consistency of the $S^{7}$ Truncation in D = 11 Supergravity. Nucl. Phys. 1987, 281, 211-240. [CrossRef]

5. Nastase, H.; Vaman, D.; van Nieuwenhuizen, P. Consistency of the $A d S_{7} \times S^{4}$ Reduction and the Origin of Selfduality in Odd Dimensions. Nucl. Phys. 2000, 581, 179-239. [CrossRef]

6. Lee, K.; Strickland-Constable, C.; Waldram, D. Spheres, generalised parallelisability and consistent truncations. Fortsch. Phys. 2017, 65, 1700048. [CrossRef]

7. Baron, W.H. Gaugings from E7(7) extended geometries. Phys. Rev. 2015, 91, 024008.

8. Hohm, O.; Samtleben, H. Consistent Kaluza-Klein Truncations via Exceptional Field Theory. J. High Energy Phys. 2015, 1, 131. [CrossRef]

9. Baron, W.H.; Dall'Agata, G. Uplifting Non-Compact Gauged Supergravities. J. High Energy Phys. 2015, 2, 3. [CrossRef]

10. Baguet, A.; Hohm, O.; Samtleben, H. Consistent Type IIB Reductions to Maximal 5D Supergravity. Phys. Rev. 2015, 92, 065004. [CrossRef]

11. Inverso, G. Generalised Scherk-Schwarz reductions from gauged supergravity. J. High Energy Phys. 2017, 12, 124. [CrossRef]

12. Ciceri, F.; de Wit, B.; Varela, O. IIB supergravity and the E6(6) covariant vector-tensor hierarchy. J. High Energy Phys. 2015, 4, 94. [CrossRef]

13. Ciceri, F.; Guarino, A.; Inverso, G. The exceptional story of massive IIA supergravity. J. High Energy Phys. 2016, 8, 154. [CrossRef]

14. Cassani, D.; de Felice, O.; Petrini, M.; Strickland-Constable, C.; Waldram, D. Exceptional Generalised Geometry for Massive IIA and Consistent Reductions. J. High Energy Phys. 2016, 8, 74. [CrossRef]

15. Guarino, A.; Jafferis, D.L.; Varela, O. String Theory Origin of Dyonic $N=8$ Supergravity and Its Chern-Simons Duals. Phys. Rev. Lett. 2015, 115, 091601. [CrossRef] [PubMed]

16. Guarino, A.; Varela, O. Dyonic ISO(7) supergravity and the duality hierarchy. J. High Energy Phys. 2016, 2, 79. [CrossRef]

17. Guarino, A.; Varela, O. Consistent $N=8$ truncation of massive IIA on $S^{6}$. J. High Energy Phys. 2015, 12, 20.

18. Malek, E. 7-dimensional $N=2$ Consistent Truncations using SL(5) Exceptional Field Theory. J. High Energy Phys. 2017, 6, 26. [CrossRef]

19. Ciceri, F.; Dibitetto, G.; Fernandez-Melgarejo, J.J.; Guarino, A.; Inverso, G. Double Field Theory at SL(2) Angles. J. High Energy Phys. 2017, 5, 28. [CrossRef]

20. Malek, E. Half-Maximal Supersymmetry from Exceptional Field Theory. Fortsch. Phys. 2017, 65, 1700061. [CrossRef]

21. Malek, E.; Samtleben, H.; Vall Camell, A. Supersymmetric $A d S_{7}$ and $A d S_{6}$ vacua and their minimal consistent truncations from exceptional field theory. Phys. Lett. 2018, 786, 171. [CrossRef]

22. Malek, E.; Samtleben, H.; Vall Camell, A. Supersymmetric $A d S_{7}$ and $A d S_{6}$ vacua and their consistent truncations with vector multiplets. J. High Energy Phys. 2019, 4, 088. [CrossRef]

23. Cassani, D.; Josse, G.; Petrini, M.; Waldram, D. Systematics of consistent truncations from generalised geometry. J. High Energy Phys. 2019, 11, 17. [CrossRef]

24. Cassani, D.; Josse, G.; Petrini, M.; Waldram, D. $N=2$ consistent truncations from wrapped M5-branes. J. High Energy Phys. 2021, 2, 232. [CrossRef]

25. Gauntlett, J.P.; Varela, O. Consistent Kaluza-Klein reductions for general supersymmetric AdS solutions. Phys. Rev. 2007, 76, 126007.

26. Gauntlett, J.P.; Kim, S.; Varela, O.; Waldram, D. Consistent Supersymmetric Kaluza-Klein Truncations with Massive Modes. J. High Energy Phys. 2009, 4, 102. [CrossRef]

27. Kashani-Poor, A.K. Nearly Kähler Reduction. J. High Energy Phys. 2007, 11, 026. [CrossRef]

28. Cassani, D.; Kashani-Poor, A.K. Exploiting N = 2 in Consistent Coset Reductions of Type IIA. Nucl. Phys. 2009, 817, 25-57. [CrossRef]

29. Cassani, D.; Dall'Agata, G.; Faedo, A.F. Type IIB Supergravity on Squashed Sasaki-Einstein Manifolds. J. High Energy Phys. 2010, 5, 94. [CrossRef]

30. Gauntlett, J.P.; Varela, O. Universal Kaluza-Klein Reductions of Type IIB to $\mathcal{N}=4$. J. High Energy Phys. 2010, 6, 81. [CrossRef]

31. Cassani, D.; Koerber, P. Tri-Sasakian Consistent Reduction. J. High Energy Phys. 2012, 1, 86. [CrossRef]

32. Cassani, D.; Koerber, P.; Varela, O. All Homogeneous N = 2 M-theory Truncations with Supersymmetric $A d S_{4}$ Vacua. J. High Energy Phys. 2012, 11, 173. [CrossRef]

33. Hull, C.M. Generalised Geometry for M-theory. J. High Energy Phys. 2007, 7, 79. [CrossRef]

34. Pires Pacheco, P.; Waldram, D. M-theory, Exceptional Generalised Geometry and Superpotentials. J. High Energy Phys. 2008, 9 , 123. [CrossRef]

35. Coimbra, A.; Strickland-Constable, C.; Waldram, D. $E_{d(d)} \times \mathbb{R}^{+}$generalised geometry, connections and M theory. J. High Energy Phys. 2014, 2, 54. [CrossRef]

36. Coimbra, A.; Strickland-Constable, C.; Waldram, D. Supergravity as Generalised Geometry II: $E_{d(d)} \times \mathbb{R}^{+}$and M theory. J. High Energy Phys. 2014, 3, 19. [CrossRef] 
37. Coimbra, A.; Strickland-Constable, C.; Waldram, D. Supersymmetric Backgrounds and Generalised Special Holonomy. Class. Quant. Grav. 2016, 33, 125026. [CrossRef]

38. De Wit, B.; Samtleben, H. Gauged Maximal Supergravities and Hierarchies of Nonabelian Vector-Tensor Systems. Fortsch. Phys. 2005, 53, 442-449. [CrossRef]

39. De Wit, B.; Nicolai, H.; Samtleben, H. Gauged Supergravities, Tensor Hierarchies, and M-theory. J. High Energy Phys. 2008, 2, 44. [CrossRef]

40. Samtleben, H. Lectures on Gauged Supergravity and Flux Compactifications. Class. Quant. Grav. 2008, 25, 214002. [CrossRef]

41. Trigiante, M. Gauged Supergravities. Phys. Rept. 2017, 680, 1-175. [CrossRef]

42. Coimbra, A.; Strickland-Constable, C. Supersymmetric Backgrounds, the Killing Superalgebra, and Generalised Special Holonomy J. High Energy Phys. 2016, 11, 63. [CrossRef]

43. Coimbra, A.; Strickland-Constable, C. Supersymmetric AdS backgrounds and weak generalised holonomy. arXiv 2017, arXiv:1710.04156.

44. Josse, G.; Malek, E.; Petrini, M.; Waldram, D. The higher-dimensional origin of five-dimensional $\mathcal{N}=2$ gauged supergravities. To appear.

45. Ashmore, A.; Waldram, D. Exceptional Calabi-Yau spaces: The geometry of $N=2$ backgrounds with flux. Fortsch. Phys. 2017, 65, 1600109. [CrossRef]

46. Schon, J.; Weidner, M. Gauged $N=4$ Supergravities. J. High Energy Phys. 2006, 5, 34. [CrossRef]

47. Günaydin, M.; Sierra, G.; Townsend, P.K. The Geometry of $N=2$ Maxwell-Einstein Supergravity and Jordan Algebras. Nucl. Phys. 1984, 242, 244. [CrossRef]

48. De Wit, B.; Vanderseypen, F.; Van Proeyen, A. Symmetry structure of special geometries. Nucl. Phys. 1993, 400, 463. [CrossRef]

49. De Wit, B.; van Zalk, M. Electric and magnetic charges in $N=2$ conformal supergravity theories. J. High Energy Phys. 2011, 10, 50. [CrossRef]

50. Louis, J.; Smyth, P.; Triendl, H. Supersymmetric Vacua in N = 2 Supergravity. J. High Energy Phys. 2012, 8, 39. [CrossRef] 